\title{
A topological degree approach to a nonlocal Neumann problem for a system at resonance
}

\author{
Katarzyna Szymańska-Dębowska and Mirosława Zima®
}

\begin{abstract}
In this paper, we investigate the existence of solutions for a system of second-order differential equations with two nonlocal Neumann boundary conditions at resonance. The existence results are obtained by applying the Mawhin continuation theorem, where the required a priori estimates are derived using some conditions of the Nirenberg type.
\end{abstract}

Mathematics Subject Classification. Primary 34B10; Secondary 34B15, $47 \mathrm{H} 10,54 \mathrm{H} 25$.

Keywords. Neumann problem, resonant problem, nonlocal boundary conditions, continuation theorem, Nirenberg conditions.

\section{Introduction}

In this paper, we consider the following nonlocal boundary value problem for the system of second-order differential equations:

$$
u_{i}^{\prime \prime}=f_{i}\left(t, u_{1}, \ldots, u_{k}\right), \quad u_{i}^{\prime}(0)=\alpha_{i}\left[u_{i}\right], \quad u_{i}^{\prime}(1)=\beta_{i}\left[u_{i}\right], i=1, \ldots, k,
$$

where the functions $f_{i}:[0,1] \times \mathbb{R}^{k} \rightarrow \mathbb{R}$ are continuous and $\alpha_{i}, \beta_{i}$ are continuous linear functionals given by the Riemann-Stieltjes integrals

$$
\alpha_{i}\left[u_{i}\right]=\int_{0}^{1} u_{i}(s) d A_{i}(s), \quad \beta_{i}\left[u_{i}\right]=\int_{0}^{1} u_{i}(s) d B_{i}(s),
$$

with the functions $A_{i}, B_{i}:[0,1] \rightarrow \mathbb{R}$ of bounded variation. Observe that problem (1.1) can be written as:

$$
u^{\prime \prime}=f(t, u), \quad u^{\prime}(0)=\alpha[u], \quad u^{\prime}(1)=\beta[u],
$$

where $u=\left(u_{1}, \ldots, u_{k}\right), f=\left(f_{1}, \ldots, f_{k}\right), \alpha[u]=\left(\alpha_{1}\left[u_{1}\right], \ldots, \alpha_{k}\left[u_{k}\right]\right)$, and $\beta[u]=\left(\beta_{1}\left[u_{1}\right], \ldots, \beta_{k}\left[u_{k}\right]\right)$. It is easy to show that problem (1.1) is at resonance if

$$
\prod_{i=1}^{k}\left(\left(1-\alpha_{i}[\gamma]\right) \beta_{i}[\delta]-\left(1-\beta_{i}[\gamma]\right) \alpha_{i}[\delta]\right)=0
$$


where $\gamma(t)=t$ and $\delta(t)=1$. This means that if (1.4) holds, then the homogeneous linear problem corresponding to (1.1), that is,

$$
u_{i}^{\prime \prime}=0, \quad u_{i}^{\prime}(0)=\alpha_{i}\left[u_{i}\right], \quad u_{i}^{\prime}(1)=\beta_{i}\left[u_{i}\right], i=1, \ldots, k,
$$

has nontrivial solutions. In particular, (1.4) is satisfied if

$$
\alpha_{i}[\delta]=\beta_{i}[\delta]=0, \quad i=1, \ldots, k .
$$

Nonlocal boundary value problems contain a class of problems involving Riemann-Stieltjes integral boundary conditions. Problems with such boundary conditions include as special cases three-point, multi-point or Riemann integral boundary conditions. Boundary value problems with nonlocal boundary conditions arise in a variety of different areas of applied mathematics and physics. They occur naturally in chemical engineering, thermo-elasticity, underground water flow, population dynamics or heat-flow problems (see, for example, $[6,11,18,22,24,25]$ and the references therein).

If in (1.2) we set $A_{i}, B_{i}=0, i=1, \ldots, k$, we obtain the classical local Neumann problem. In the local case, the Neumann problems have been widely studied by a number of authors (see, e.g., [3-5, 7, 9, 10, 13, 14]). In particular, recent existence and localization results on positive solutions for such problems can be found in $[5,7,10,25]$. Recently, the scalar Neumann problems have also been studied in [11]. For both local and nonlocal nonresonant case, the authors obtained results on the existence, non-existence, localization and multiplicity of nontrivial solutions by dealing with a suitable perturbed Hammerstein integral equation. Their tool was the fixed point index for compact operators in cones. Other types of nonlocal Neumann problems than those discussed in this paper can be found, for instance, in $[16,21,23]$.

Our main goal in this article is to establish sufficient conditions for the existence of a solution to problem (1.1) at resonance. More precisely, we focus on the case the condition (1.5) is satisfied. We point out that, in contrast to the papers [16] and [23], both the boundary conditions are nonlocal. To provide the main results of the paper, we apply the celebrated Mawhin continuation theorem (see, for example, $[8,15]$ ). The key idea is to write problem (1.1) as a semilinear equation $L u=N u$, where $L$ is a linear Fredholm operator of index zero and $N$ is a nonlinear map, and to derive a priori estimates for its possible solutions and their derivatives. The estimates are obtained by imposing upon nonlinearity $f$ some conditions of the Nirenberg type. Such conditions were introduced in 1971 by Nirenberg [17] in the context of elliptic boundary value problems, generalizing the one introduced in 1970 by Landesman and Lazer [12] for semilinear elliptic equations with resonant linear part. For a detailed exposition and applications, see for example [1,16,21,23].

It is worth noting that the Mawhin continuation theorem was previously used to prove existence theorems for the local Neumann problems at resonance in $[2,19,20]$. In particular, in [19] the author dealt with the scalar problem

$$
x^{\prime \prime}=f\left(t, x, x^{\prime}\right), \quad x^{\prime}(a)=0, \quad x^{\prime}(b)=0 .
$$


The existence and multiplicity results have been proved by means of the connection between the topological degree of the operator associated with (1.6) and strict lower and upper solutions of (1.6).

The paper is organized as follows. In Sect. 2, we present some preparatory material concerning re-writing the resonant problem (1.3) in the setting of semilinear equation. Section 3 deals with the main results of the paper, namely Theorems 3.1 and 3.4. We then give some examples of a class of functions, for which the Nirenberg-type conditions hold (Corollaries 3.2 and 3.3). Finally, Sect. 4 is dedicated to discussing some particular Neumann problems related to (1.3).

\section{Preliminaries}

Denote by $\langle\cdot, \cdot\rangle$ the standard inner product in $\mathbb{R}^{k}$ and by $|\cdot|$ the corresponding Euclidean norm in $\mathbb{R}^{k}$. Moreover, let us consider the Banach space $C^{1}\left([0,1], \mathbb{R}^{k}\right)$ of all continuously differentiable functions $u:[0,1] \rightarrow \mathbb{R}^{k}$ equipped with the norm

$$
\|u\|=\max \left\{\|u\|_{\infty},\left\|u^{\prime}\right\|_{\infty}\right\},
$$

where $\|\cdot\|_{\infty}$ stands for the supremum norm in $C\left([0,1], \mathbb{R}^{k}\right)$. We will work in the Banach space

$$
U=\left\{u \in C^{1}\left([0,1], \mathbb{R}^{k}\right): u^{\prime}(0)=\alpha[u], \quad u^{\prime}(1)=\beta[u]\right\}
$$

with the norm (2.1).

Let us introduce the notation:

$$
\begin{aligned}
k(t, s) & = \begin{cases}t-s, & 0 \leq s \leq t \leq 1 \\
0, & 0 \leq t \leq s \leq 1\end{cases} \\
\mathcal{K}_{A_{i}}(s) & =\int_{0}^{1} k(t, s) d A_{i}(t), \mathcal{K}_{B_{i}}(s)=\int_{0}^{1} k(t, s) d B_{i}(t), \quad i=1, \ldots, k .
\end{aligned}
$$

Throughout the paper, we will make use of the following assumptions:

(H1) $f:[0,1] \times \mathbb{R}^{k} \rightarrow \mathbb{R}^{k}$ is continuous and bounded;

$(\mathrm{H} 2) A, B:[0,1] \rightarrow \mathbb{R}^{k}, A:=\left(A_{1}, \ldots, A_{k}\right), B:=\left(B_{1}, \ldots, B_{k}\right)$ have bounded variation, and $\alpha_{i}[\delta]=\beta_{i}[\delta]=0$ for $i=1, \ldots, k$;

(H3) $\alpha_{i}[\gamma] \neq 1$ and $\int_{0}^{1}\left[\mathcal{K}_{B_{i}}(s)-\left(1-\alpha_{i}[\gamma]\right)^{-1}\left(1-\beta_{i}[\gamma]\right) \mathcal{K}_{A_{i}}(s)\right] \mathrm{d} s \neq 1$ for $i=1, \ldots, k$;

(H4) $\beta_{i}[\gamma] \neq 1$ and $\int_{0}^{1}\left[\left(1-\alpha_{i}[\gamma]\right)\left(1-\beta_{i}[\gamma]\right)^{-1}\left(\mathcal{K}_{B_{i}}(s)-1\right)-\mathcal{K}_{A_{i}}(s)\right] \mathrm{d} s \neq$ 0 for $i=1, \ldots, k$.

To transform (1.3) into a semilinear equation, we define the linear mapping $L: \operatorname{Dom} L \rightarrow C\left([0,1], \mathbb{R}^{k}\right)$ by setting

$$
\operatorname{Dom} L:=\left\{u \in U: u^{\prime \prime} \in C\left([0,1], \mathbb{R}^{k}\right)\right\}
$$

and for $u \in \operatorname{Dom} L$,

$$
L u=u^{\prime \prime} .
$$


Observe that

$$
\operatorname{Ker} L=\left\{u \in \operatorname{Dom} L: u=c \in \mathbb{R}^{k}\right\}=\mathbb{R}^{k} .
$$

Thus, $\operatorname{dim} \operatorname{Ker} L=k$.

Assume now that (H2) and (H3) are satisfied. If $y=\left(y_{1}, \ldots, y_{k}\right) \in \operatorname{Im} L$, then there is a $u=\left(u_{1}, \ldots, u_{k}\right) \in \operatorname{Dom} L$ such that $u_{i}^{\prime \prime}=y_{i}, i=1, \ldots, k$. Integrating twice this equation from 0 to $t$, one has

$$
u_{i}^{\prime}(t)=u_{i}^{\prime}(0)+\int_{0}^{t} y_{i}(s) \mathrm{d} s
$$

and

$$
u_{i}(t)=u_{i}(0)+u_{i}^{\prime}(0) t+\int_{0}^{1} k(t, s) y_{i}(s) \mathrm{d} s,
$$

$i=1, \ldots, k$. Since $u_{i}^{\prime}(0)=\alpha_{i}\left[u_{i}\right]$, we obtain from (H2), (H3), and (2.3)

$$
u_{i}^{\prime}(0)=\left(1-\alpha_{i}[\gamma]\right)^{-1} \int_{0}^{1} \mathcal{K}_{A_{i}}(s) y_{i}(s) \mathrm{d} s .
$$

Similarly, from $u_{i}^{\prime}(1)=\beta_{i}\left[u_{i}\right]$, we have

$$
\begin{gathered}
\left(1-\alpha_{i}[\gamma]\right)^{-1}\left(1-\beta_{i}[\gamma]\right) \int_{0}^{1} \mathcal{K}_{A_{i}}(s) y_{i}(s) \mathrm{d} s \\
+\int_{0}^{1} y_{i}(s) \mathrm{d} s-\int_{0}^{1} \mathcal{K}_{B_{i}}(s) y_{i}(s) \mathrm{d} s=0 .
\end{gathered}
$$

For every $a, b \in \mathbb{R}^{k}$, we define $a \cdot b:=\left(a_{1} b_{1}, \ldots, a_{k} b_{k}\right)$. We also write $a^{-1}:=\left(\frac{1}{a_{1}}, \ldots, \frac{1}{a_{k}}\right)$ provided that $a_{i} \neq 0$ for $i=1, \ldots, k$. Moreover, we set $\mathcal{K}_{A}(s)=\left(\mathcal{K}_{A_{1}}(s), \ldots, \mathcal{K}_{A_{k}}(s)\right)$ and $\mathcal{K}_{B}(s)=\left(\mathcal{K}_{B_{1}}(s), \ldots, \mathcal{K}_{B_{k}}(s)\right)$. Then, condition (2.4) takes the following form:

$$
\begin{gathered}
(1-\alpha[\gamma])^{-1} \cdot(1-\beta[\gamma]) \cdot \int_{0}^{1} \mathcal{K}_{A}(s) \cdot y(s) \mathrm{d} s \\
+\int_{0}^{1} y(s) \mathrm{d} s-\int_{0}^{1} \mathcal{K}_{B}(s) \cdot y(s) \mathrm{d} s=0 .
\end{gathered}
$$

Consequently, we obtain

$$
\begin{aligned}
\operatorname{Im} L:= & \left\{y \in C\left([0,1], \mathbb{R}^{k}\right):(1-\alpha[\gamma])^{-1} \cdot(1-\beta[\gamma]) \cdot \int_{0}^{1} \mathcal{K}_{A}(s) \cdot y(s) \mathrm{d} s\right. \\
& \left.+\int_{0}^{1} y(s) \mathrm{d} s-\int_{0}^{1} \mathcal{K}_{B}(s) \cdot y(s) \mathrm{d} s=0\right\} .
\end{aligned}
$$

Hence, for every $y \in \operatorname{Im} L$ we have $L u=y$, where $u \in \operatorname{Dom} L$ and

$$
u(t)=u(0)+t(1-\alpha[\gamma])^{-1} \cdot \int_{0}^{1} \mathcal{K}_{A}(s) \cdot y(s) \mathrm{d} s+\int_{0}^{1} k(t, s) y(s) \mathrm{d} s .
$$


Assumption (H3) implies that $C\left([0,1], \mathbb{R}^{k}\right)=\tilde{Y} \oplus \operatorname{Im} L$, where

$$
\begin{aligned}
\widetilde{Y}= & \left\{\widetilde{y} \in C\left([0,1], \mathbb{R}^{k}\right): \widetilde{y}=\widetilde{M} \cdot\left((1-\alpha[\gamma])^{-1} \cdot(1-\beta[\gamma])\right.\right. \\
& \left.\left.\cdot \int_{0}^{1} \mathcal{K}_{A}(s) \cdot y(s) \mathrm{d} s+\int_{0}^{1} y(s) \mathrm{d} s-\int_{0}^{1} \mathcal{K}_{B}(s) \cdot y(s) \mathrm{d} s\right), y \in C\left([0,1], \mathbb{R}^{k}\right)\right\}
\end{aligned}
$$

with

$$
\widetilde{M}:=\left(\int_{0}^{1}\left((1-\alpha[\gamma])^{-1} \cdot(1-\beta[\gamma]) \cdot \mathcal{K}_{A}(s)-\mathcal{K}_{B}(s)\right) \mathrm{d} s+\widehat{1}\right)^{-1} .
$$

Here, $\hat{1}$ denotes the constant vector $(1,1, \ldots, 1) \in \mathbb{R}^{k}$. Thus, $\operatorname{Im} L$ is closed and $\operatorname{codim} \operatorname{Im} L=k=\operatorname{dim} \operatorname{Ker} L$. This means that $L$ is a Fredholm operator of index 0. Moreover, $\operatorname{Ker} L=\operatorname{Im} P$ and $\operatorname{Im} L=\operatorname{Ker} Q$, where $P$ is the projector in $U$ defined by $P u=u(0)$, and $Q$ is the projector in $C\left([0,1], \mathbb{R}^{k}\right)$ defined by

$$
\begin{aligned}
Q y= & \widetilde{M} \cdot\left((1-\alpha[\gamma])^{-1} \cdot(1-\beta[\gamma]) \cdot \int_{0}^{1} \mathcal{K}_{A}(s) \cdot y(s) \mathrm{d} s\right. \\
& \left.+\int_{0}^{1} y(s) \mathrm{d} s-\int_{0}^{1} \mathcal{K}_{B}(s) \cdot y(s) \mathrm{d} s\right) .
\end{aligned}
$$

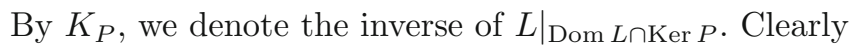

$$
K_{P} y(t)=t(1-\alpha[\gamma])^{-1} \cdot \int_{0}^{1} \mathcal{K}_{A}(s) \cdot y(s) \mathrm{d} s+\int_{0}^{1} k(t, s) y(s) \mathrm{d} s, \quad t \in[0,1]
$$

Now, let $\Omega$ be an open bounded subset of $U$. We define the nonlinear mapping $N: \bar{\Omega} \rightarrow C\left([0,1], \mathbb{R}^{k}\right)$ by

$$
N u(t)=f(t, u(t)) .
$$

It is easy to show that (H1) and (H2) imply that $N$ is continuous and $L$-compact on $\bar{\Omega}$, that is, $Q N(\bar{\Omega})$ is bounded and $K_{P}(I-Q) N: \bar{\Omega} \rightarrow$ $C\left([0,1], \mathbb{R}^{k}\right)$ is compact. By the above, problem (1.3) is equivalent to the abstract equation

$$
L u=N u, \quad u \in \operatorname{Dom} L .
$$

Let $J: \operatorname{Im} Q \rightarrow$ Ker $L$ be such that $J(a)=a, a \in \mathbb{R}^{k}$. Taking into account the above considerations, the Mawhin continuation theorem $[8, \mathrm{p}$. 40] can be written as follows:

Lemma 2.1. Assume that (H1)-(H3) are satisfied. Let $\Omega$ be an open and bounded set in $U$ such that the following conditions hold:

(M1) For each $\lambda \in(0,1)$, the equation

$$
u^{\prime \prime}=\lambda f(t, u)
$$

has no solution on $\partial \Omega \cap \operatorname{Dom} L$; 
(M2) The equation

$$
\begin{aligned}
F(a):= & (1-\alpha[\gamma])^{-1} \cdot(1-\beta[\gamma]) \cdot \int_{0}^{1} \mathcal{K}_{A}(s) \cdot f(s, a) \mathrm{d} s \\
& +\int_{0}^{1} f(s, a) \mathrm{d} s-\int_{0}^{1} \mathcal{K}_{B}(s) \cdot f(s, a) \mathrm{d} s=0
\end{aligned}
$$

has no solution on $\partial \Omega \cap \mathbb{R}^{k}$;

(M3) $\operatorname{deg}_{\mathrm{B}}\left(F, \Omega \cap \mathbb{R}^{k}, 0\right) \neq 0$, where $\operatorname{deg}_{\mathrm{B}}$ stands for the Brouwer degree.

Then, problem (1.3) has a solution in $\bar{\Omega}$.

Now let us assume that (H4) holds instead of (H3). The following result can be obtained in the same way as Lemma 2.1 .

Lemma 2.2. Assume that (H1), (H2), and (H4) are satisfied. Let $\Omega$ be an open and bounded set in $U$ such that (M1) holds, and moreover:

(M2*) The equation

$$
\begin{aligned}
F^{*}(a):= & (1-\alpha[\gamma]) \cdot(1-\beta[\gamma])^{-1} \cdot\left(\int_{0}^{1} \mathcal{K}_{B}(s) \cdot f(s, a) \mathrm{d} s\right. \\
& \left.-\int_{0}^{1} f(s, a) \mathrm{d} s\right)-\int_{0}^{1} \mathcal{K}_{A}(s) \cdot f(s, a) \mathrm{d} s=0
\end{aligned}
$$

has no solution on $\partial \Omega \cap \mathbb{R}^{k}$;

$\left(M 3^{*}\right) \operatorname{deg}_{\mathrm{B}}\left(F^{*}, \Omega \cap \mathbb{R}^{k}, 0\right) \neq 0$.

Then, problem (1.3) has a solution in $\bar{\Omega}$.

\section{Existence results}

In this section, we derive the main existence results for solutions to problem (1.3). Let us introduce the following assumption upon $f$ :

(G1) For every $t \in[0,1]$, there exists a uniform finite limit

$$
g(t, \xi):=\lim _{\lambda \rightarrow \infty} f(t, \lambda \xi)
$$

with respect to $\xi \in S^{k-1}$, with $S^{k-1}$ the unit sphere in $\mathbb{R}^{k}$.

We use $B(0, r)$ and $\bar{B}(0, r)$ to denote the open and, respectively, the closed ball in $\mathbb{R}^{k}$ with the center at 0 and the radius $r$.

Let us recall that if $\varphi: S^{k-1} \rightarrow S^{k-1}$ is continuous, $0 \notin \varphi\left(S^{k-1}\right)$ and $\bar{\varphi}: \bar{B}(0,1) \rightarrow \mathbb{R}^{k}$ is any continuous extension of $\varphi$, then the degree of $\varphi$ is defined as:

$$
\operatorname{deg}_{\mathrm{B}}(\varphi):=\operatorname{deg}_{\mathrm{B}}(\bar{\varphi}, B(0,1), 0) .
$$

Theorem 3.1. Let assumptions (H1)-(H3), and (G1) be satisfied. For $\xi \in$ $S^{k-1}$ set

$$
\begin{aligned}
G(\xi):= & (1-\alpha[\gamma])^{-1} \cdot(1-\beta[\gamma]) \cdot \int_{0}^{1} \mathcal{K}_{A}(s) \cdot g(s, \xi) \mathrm{d} s \\
& +\int_{0}^{1} g(s, \xi) \mathrm{d} s-\int_{0}^{1} \mathcal{K}_{B}(s) \cdot g(s, \xi) \mathrm{d} s .
\end{aligned}
$$


Then, problem (1.3) has at least one solution if the following conditions hold: (G2) $G(\xi) \neq 0$ for each $\xi \in S^{k-1}$;

(G3) $\operatorname{deg}_{\mathrm{B}}(\varphi) \neq 0$, where $\varphi: S^{k-1} \rightarrow S^{k-1}$ is the mapping defined by

$$
\varphi(\xi)=\frac{G(\xi)}{|G(\xi)|}
$$

Proof. For the proof, we shall use Lemma 2.1. First, we shall show that all solutions to equation (2.5) are bounded, when $\lambda \in(0,1)$ and $u \in \operatorname{Dom} L$. Suppose, on the contrary, that

$$
u_{n}^{\prime \prime}=\lambda_{n} f\left(t, u_{n}\right), \quad u_{n}^{\prime}(0)=\alpha\left[u_{n}\right], \quad u_{n}^{\prime}(1)=\beta\left[u_{n}\right],
$$

for some $\lambda_{n} \in(0,1)$ and the sequence $\left(u_{n}\right)$ is unbounded. Then, passing to a subsequence if necessary, we have $\left\|u_{n}\right\| \rightarrow \infty, n \rightarrow \infty$.

From (3.1), one has

$$
\begin{aligned}
u_{n}^{\prime}(t)= & \lambda_{n}(1-\alpha[\gamma])^{-1} \cdot \int_{0}^{1} \mathcal{K}_{A}(s) \cdot f\left(s, u_{n}(s)\right) \mathrm{d} s \\
& +\lambda_{n} \int_{0}^{t} f\left(s, u_{n}(s)\right) \mathrm{d} s .
\end{aligned}
$$

By the assumption (H1), the function $f$ is bounded. Thus, by (3.2), we get that there is $M>0$ such that $\left\|u_{n}^{\prime}\right\|_{\infty} \leq M$, for every $n$. Hence

$$
\left\|u_{n}\right\|_{\infty} \rightarrow \infty
$$

when $n \rightarrow \infty$.

Now, let us consider in the space $C^{1}\left([0,1], \mathbb{R}^{k}\right)$ the sequence

$$
v_{n}:=\frac{u_{n}}{\left\|u_{n}\right\|} .
$$

Clearly, $\left\|v_{n}\right\|=1$ for every $n \in \mathbb{N}$. Hence, the sequence $\left(v_{n}\right)$ is bounded. It is easy to observe that the families $\left(v_{n}\right)$ and $\left(v_{n}^{\prime}\right)$ are uniformly bounded and equicontinuous (see [23]). Using the Ascoli-Arzelà theorem, we conclude that there exists a convergent subsequence of $\left(v_{n}\right)$, which we also denote by $\left(v_{n}\right)$. By (3.1) and (3.2), we obtain

$$
\begin{aligned}
u_{n}(t)= & u_{n}(0)+\lambda_{n} t(1-\alpha[\gamma])^{-1} \cdot \int_{0}^{1} \mathcal{K}_{A}(s) \cdot f\left(s, u_{n}(s)\right) \mathrm{d} s \\
& +\lambda_{n} \int_{0}^{1} k(t, s) f\left(s, u_{n}(s)\right) \mathrm{d} s .
\end{aligned}
$$

Note that the sequence $\left(v_{n}\right)$ converges uniformly to a $\xi \in S^{k-1}$ on $[0,1]$. Indeed, since $f$ is bounded, the sequence

$$
\left(\frac{\lambda_{n} t(1-\alpha[\gamma])^{-1} \cdot \int_{0}^{1} \mathcal{K}_{A}(s) \cdot f\left(s, u_{n}(s)\right) \mathrm{d} s+\lambda_{n} \int_{0}^{1} k(t, s) f\left(s, u_{n}(s)\right) \mathrm{d} s}{\left\|u_{n}\right\|}\right)
$$

tends to $0 \in \mathbb{R}^{k}$ uniformly on $[0,1]$. Thus, by (3.3), one gets that the limit of $\left(v_{n}\right)$ does not depend on $t$. Consequently, since for every $n \in \mathbb{N},\left\|v_{n}\right\|=1$ and the sequence $\left(v_{n}\right)$ is convergent, we infer, without using the formula (3.3), that $\left(v_{n}\right)$ tends to a $\xi \in S^{k-1}$ and this convergence is uniform (see also [23, 
Lemma 4]). Moreover, it can be shown that $f\left(t,\left\|u_{n}\right\| \frac{u_{n}(t)}{\left\|u_{n}\right\|}\right)$ tends to $g(t, \xi)$ for each $t \in[0,1]$ ( see [23, Lemma 5]).

It is easily verified that integration of the equation in (3.1) and the boundary conditions yield

$$
\begin{aligned}
& (1-\alpha[\gamma])^{-1} \cdot(1-\beta[\gamma]) \cdot \int_{0}^{1} \mathcal{K}_{A}(s) \cdot f\left(s, u_{n}(s)\right) \mathrm{d} s \\
& \quad+\int_{0}^{1} f\left(s, u_{n}(s)\right) \mathrm{d} s-\int_{0}^{1} \mathcal{K}_{B}(s) \cdot f\left(s, u_{n}(s)\right) \mathrm{d} s=0
\end{aligned}
$$

for every $n \in \mathbb{N}$. On the other hand, by assumption (G2) we reach the required contradiction since

$$
\begin{aligned}
\lim _{n \rightarrow \infty} & \left((1-\alpha[\gamma])^{-1} \cdot(1-\beta[\gamma]) \cdot \int_{0}^{1} \mathcal{K}_{A}(s) \cdot f\left(s, u_{n}(s)\right) \mathrm{d} s\right. \\
& \left.+\int_{0}^{1} f\left(s, u_{n}(s)\right) \mathrm{d} s-\int_{0}^{1} \mathcal{K}_{B}(s) \cdot f\left(s, u_{n}(s)\right) \mathrm{d} s\right) \\
= & \lim _{n \rightarrow \infty}\left((1-\alpha[\gamma])^{-1} \cdot(1-\beta[\gamma]) \cdot \int_{0}^{1} \mathcal{K}_{A}(s) \cdot f\left(s,\left\|u_{n}\right\| \frac{u_{n}(s)}{\left\|u_{n}\right\|}\right) \mathrm{d} s\right. \\
& \left.+\int_{0}^{1} f\left(s,\left\|u_{n}\right\| \frac{u_{n}(s)}{\left\|u_{n}\right\|}\right) \mathrm{d} s-\int_{0}^{1} \mathcal{K}_{B}(s) \cdot f\left(s,\left\|u_{n}\right\| \frac{u_{n}(s)}{\left\|u_{n}\right\|}\right) \mathrm{d} s\right) \\
= & (1-\alpha[\gamma])^{-1} \cdot(1-\beta[\gamma]) \cdot \int_{0}^{1} \mathcal{K}_{A}(s) \cdot g(s, \xi) \mathrm{d} s \\
& +\int_{0}^{1} g(s, \xi) \mathrm{d} s-\int_{0}^{1} \mathcal{K}_{B}(s) \cdot g(s, \xi) \mathrm{d} s \neq 0 .
\end{aligned}
$$

Consequently, all solutions to problem (2.5) are bounded for $\lambda \in(0,1)$ and $u \in \operatorname{Dom} L$.

Now, assumption (G2) implies that there exists $R_{0}>0$ such that $F$ defined by (2.6) does not vanish outside a ball $B\left(0, R_{0}\right)$, so by the excision property of the Brouwer degree, $\operatorname{deg}_{\mathrm{B}}(F, B(0, R), 0)$ is well defined, independent of $R, R \geq R_{0}$, and

$$
\operatorname{deg}_{\mathrm{B}}\left(F, B\left(0, R_{0}\right), 0\right)=\operatorname{deg}_{\mathrm{B}}(F, B(0, R), 0) .
$$

Let $\varphi_{R}: S^{k-1} \rightarrow S^{k-1}$ be given by

$$
\varphi_{R}(\xi)=\frac{F(R \xi)}{|F(R \xi)|} .
$$

Observe that the normalized mapping $\varphi$ defined in (G3) is continuous. From assumptions (G1), (G2) and the Lebesgue dominated convergence theorem,

$$
\lim _{R \rightarrow \infty} F(R \xi)=G(\xi) \neq 0,
$$

uniformly on $S^{k-1}$, which implies that $\varphi_{R} \rightarrow \varphi$ uniformly. By the invariance of the Brouwer degree under small perturbations, there exists $\widetilde{R} \geq R_{0}$ such that $\operatorname{deg}_{\mathrm{B}}\left(\varphi_{\widetilde{R}}\right)=\operatorname{deg}_{\mathrm{B}}(\varphi)$. 
We now want to show that $\operatorname{deg}_{\mathrm{B}}(F, B(0, \widetilde{R}), 0) \neq 0$. To this end, for $\mu \in[0,1]$ and $\xi \in S^{k-1}$ consider the following homotopy

$$
\mathcal{H}(\mu, \xi)=\mu \varphi_{\widetilde{R}}(\xi)+(1-\mu) F(\widetilde{R} \xi) .
$$

By (G2), we have $\mathcal{H}(\mu, \xi) \neq 0$ for $\mu \in[0,1]$ and $\xi \in S^{k-1}$. Thus, from the homotopy invariance of the Brouwer degree, one has

$$
\operatorname{deg}_{\mathrm{B}}\left(\varphi_{\widetilde{R}}\right)=\operatorname{deg}_{\mathrm{B}}(F(\widetilde{R} \cdot))=\operatorname{deg}_{\mathrm{B}}(F, B(0, \widetilde{R}), 0) .
$$

Therefore, by (G3), we obtain

$$
0 \neq \operatorname{deg}_{\mathrm{B}}(\varphi)=\operatorname{deg}_{\mathrm{B}}\left(\varphi_{\widetilde{R}}\right)=\operatorname{deg}_{\mathrm{B}}(F, B(0, \widetilde{R}), 0),
$$

which completes the proof.

Let us mention that the assumptions (G2) and (G3) may be in general difficult to verify. We now give two sufficient conditions, formulated in terms of the inner product in $\mathbb{R}^{k}$, that imply (G2) and (G3) and can be easier to deal with.

Corollary 3.2. Let assumptions (H1)-(H3), and (G1) hold. Moreover, let

$$
\langle\xi, G(\xi)\rangle<0
$$

for all $\xi \in S^{k-1}$. Then, problem (1.3) has a solution.

Corollary 3.3. Let assumptions (H1)-(H3), and (G1) be satisfied. Moreover, let

$$
\langle\xi, G(\xi)\rangle>0
$$

for all $\xi \in S^{k-1}$. Then, there exists a solution to problem (1.3). results.

In the case when (H4) holds instead of (H3), we obtain the following

Theorem 3.4. Let assumptions (H1), (H2), (H4), and (G1) be satisfied. If (G2) and (G3) of Theorem 3.1 hold with

$$
\begin{aligned}
G(\xi):= & (1-\alpha[\gamma]) \cdot(1-\beta[\gamma])^{-1} \cdot\left(\int_{0}^{1} \mathcal{K}_{B}(s) \cdot g(s, \xi) \mathrm{d} s\right. \\
& \left.-\int_{0}^{1} g(s, \xi) \mathrm{d} s\right)-\int_{0}^{1} \mathcal{K}_{A}(s) \cdot g(s, \xi) \mathrm{d} s
\end{aligned}
$$

then there exists a solution to problem (1.3).

Proof. The proof is analogous to the one of Theorem 3.1. Here, one should apply Lemma 2.2.

Corollary 3.5. Let assumptions (H1), (H2), (H4), and (G1) hold. Moreover, assume that the function $G$ defined by (3.6) satisfies either (3.4) or (3.5). Then, problem (1.3) has a solution.

We complete this section with an example illustrating our theoretical results. 
Example. Consider problem (1.3) with

$$
\begin{aligned}
& f_{1}\left(t, u_{1}, u_{2}\right)=\frac{t u_{1}+\sin \left(t^{2}-u_{1} u_{2}\right)}{1+t+\sqrt{u_{1}^{2}+u_{2}^{2}}}, \\
& f_{2}\left(t, u_{1}, u_{2}\right)=\frac{e^{t} u_{2}^{3}}{\sqrt{u_{1}^{6}+u_{2}^{6}+1}},
\end{aligned}
$$

$\alpha_{1}\left[u_{1}\right]=u_{1}\left(\frac{3}{4}\right)-u_{1}\left(\frac{1}{4}\right), \alpha_{2}\left[u_{2}\right]=u_{2}(1)-u_{2}\left(\frac{1}{2}\right), \beta_{1}\left[u_{1}\right]=\int_{0}^{1} u_{1}(t) \cos \pi t \mathrm{~d} t$, and $\beta_{2}\left[u_{2}\right]=\int_{0}^{1} u_{2}(t)\left(\frac{1}{2}-t\right) \mathrm{d} t$. It is clear that

$$
\begin{aligned}
& A_{1}(t)=\left\{\begin{array}{rr}
0, & t \in\left[0, \frac{1}{4}\right] \cup\left[\frac{3}{4}, 1\right] \\
-1, & t \in\left(\frac{1}{4}, \frac{3}{4}\right)
\end{array},\right. \\
& A_{2}(t)=\left\{\begin{array}{rr}
0, & t \in\left[0, \frac{1}{2}\right] \cup\{1\} \\
-1, & t \in\left(\frac{1}{2}, 1\right)
\end{array}\right. \\
& B_{1}(t)=\frac{1}{\pi} \sin \pi t
\end{aligned}
$$

and

$$
B_{2}(t)=\frac{1}{2} t-\frac{1}{2} t^{2}
$$

Moreover, $\alpha_{1}[\delta]=\alpha_{2}[\delta]=\beta_{1}[\delta]=\beta_{2}[\delta]=0$, so the problem is at resonance. Furthermore, we have $\alpha_{1}[\gamma]=\alpha_{2}[\gamma]=\frac{1}{2}, \beta_{1}[\gamma]=-\frac{2}{\pi^{2}}, \beta_{2}[\gamma]=-\frac{1}{12}$,

$$
\begin{aligned}
& \mathcal{K}_{A_{1}}(s)=\left\{\begin{array}{rr}
\frac{1}{2}, & 0 \leq s<\frac{1}{4} \\
\frac{3}{4}-s, & \frac{1}{4} \leq s<\frac{3}{4} \\
0, & \frac{3}{4} \leq s \leq 1
\end{array}\right. \\
& \mathcal{K}_{A_{2}}(s)=\left\{\begin{array}{rr}
\frac{1}{2}, & 0 \leq s<\frac{1}{2} \\
1-s, & \frac{1}{2} \leq s \leq 1
\end{array}\right. \\
& \mathcal{K}_{B_{1}}(s)=-\frac{1}{\pi^{2}}(1+\cos \pi s)
\end{aligned}
$$

and

$$
\mathcal{K}_{B_{2}}(s)=-\frac{1}{12}+\frac{1}{4} s^{2}-\frac{1}{6} s^{3} .
$$

By a direct calculation, we get

$$
\int_{0}^{1}\left[\mathcal{K}_{B_{1}}(s)-\left(1-\alpha_{1}[\gamma]\right)^{-1}\left(1-\beta_{1}[\gamma]\right) \mathcal{K}_{A_{1}}(s)\right] \mathrm{d} s=-\frac{1}{2}-\frac{2}{\pi^{2}}
$$

and

$$
\int_{0}^{1}\left[\mathcal{K}_{B_{2}}(s)-\left(1-\alpha_{2}[\gamma]\right)^{-1}\left(1-\beta_{2}[\gamma]\right) \mathcal{K}_{A_{2}}(s)\right] \mathrm{d} s=-\frac{41}{48} .
$$

Thus, the assumptions (H1)-(H3) hold. Since for $t \in[0,1]$ and $\left(\xi_{1}, \xi_{2}\right) \in S^{1}$, we have

$$
g_{1}\left(t, \xi_{1}, \xi_{2}\right)=\lim _{\lambda \rightarrow \infty} f_{1}\left(t, \lambda \xi_{1}, \lambda \xi_{2}\right)=t \xi_{1}
$$


and

$$
g_{2}\left(t, \xi_{1}, \xi_{2}\right)=\lim _{\lambda \rightarrow \infty} f_{2}\left(t, \lambda \xi_{1}, \lambda \xi_{2}\right)=\frac{e^{t} \xi_{2}^{3}}{\sqrt{\xi_{1}^{6}+\xi_{2}^{6}}},
$$

assumption (G1) is also satisfied. Moreover, for $\left(\xi_{1}, \xi_{2}\right) \in S^{1}$

$$
\begin{aligned}
G_{1}\left(\xi_{1}, \xi_{2}\right)= & \left(1-\alpha_{1}[\gamma]\right)^{-1}\left(1-\beta_{1}[\gamma]\right) \int_{0}^{1} \mathcal{K}_{A_{1}}(s) g_{1}\left(s, \xi_{1}, \xi_{2}\right) \mathrm{d} s \\
& +\int_{0}^{1} g_{1}\left(s, \xi_{1}, \xi_{2}\right) \mathrm{d} s-\int_{0}^{1} \mathcal{K}_{B_{1}}(s) g_{1}\left(s, \xi_{1}, \xi_{2}\right) \mathrm{d} s \\
= & \xi_{1}\left[\left(2+\frac{4}{\pi^{2}}\right) \int_{0}^{1} \mathcal{K}_{A_{1}}(s) s \mathrm{~d} s+\frac{1}{2}-\int_{0}^{1} \mathcal{K}_{B_{1}}(s) s \mathrm{~d} s\right]
\end{aligned}
$$

and

$$
\begin{aligned}
G_{2}\left(\xi_{1}, \xi_{2}\right)= & \left(1-\alpha_{2}[\gamma]\right)^{-1}\left(1-\beta_{2}[\gamma]\right) \int_{0}^{1} \mathcal{K}_{A_{2}}(s) g_{2}\left(s, \xi_{1}, \xi_{2}\right) \mathrm{d} s \\
& +\int_{0}^{1} g_{2}\left(s, \xi_{1}, \xi_{2}\right) \mathrm{d} s-\int_{0}^{1} \mathcal{K}_{B_{2}}(s) g_{2}\left(s, \xi_{1}, \xi_{2}\right) \mathrm{d} s \\
= & \frac{\xi_{2}^{3}}{\sqrt{\xi_{1}^{6}+\xi_{2}^{6}}}\left[\frac{13}{6} \int_{0}^{1} \mathcal{K}_{A_{2}}(s) e^{s} \mathrm{~d} s+e-1-\int_{0}^{1} \mathcal{K}_{B_{2}}(s) e^{s} \mathrm{~d} s\right] .
\end{aligned}
$$

Observe that $\mathcal{K}_{A_{i}}(s) \geq 0$ and $\mathcal{K}_{B_{i}}(s) \leq 0$. Therefore, (3.5) is satisfied. By Corollary 3.3 , there exists a nontrivial solution to problem (1.3).

\section{Final remarks}

Remark 4.1. Let $k=1$ and let us consider problem (1.3) in the following form:

$$
u^{\prime \prime}=f(u), \quad u^{\prime}(0)=\alpha[u], \quad u^{\prime}(1)=\beta[u]
$$

Set

$$
f( \pm \infty)=\lim _{u \rightarrow \pm \infty} f(u)
$$

Note that then the conditions (3.4) and (3.5) are given by

$$
f(+\infty)<0<f(-\infty)
$$

and

$$
f(-\infty)<0<f(+\infty)
$$

respectively. Consequently, when the nonlinearity $f$ depends only on $u$, we obtain the classical Landesman-Lazer conditions [12]. It is also interesting to note that if either (4.1) or (4.2) holds, then problem (1.3) has at least one constant solution.

Remark 4.2. Observe that in the case of the local Neumann boundary conditions, that is, when $A_{i}=B_{i}=0$ for $i=1, \ldots, k$, the assumptions (H2)-(H4) are satisfied. Moreover, $G(\xi)=\int_{0}^{1} g(s, \xi) \mathrm{d} s$ for $\xi \in S^{k-1}$, so (G2) now reads 
$\int_{0}^{1} g(s, \xi) \mathrm{d} s \neq 0$ and $(\mathrm{G} 3)$ becomes $\operatorname{deg}_{\mathrm{B}}(\varphi) \neq 0$, where $\varphi: S^{k-1} \rightarrow S^{k-1}$ is given by

$$
\varphi(\xi)=\frac{\int_{0}^{1} g(s, \xi) \mathrm{d} s}{\left|\int_{0}^{1} g(s, \xi) \mathrm{d} s\right|} .
$$

Thus, in this special case, Theorem 3.1 corresponds to some extent to Theorem 1.2 of [2], which deals with the local problem

$$
\left\{\begin{array}{l}
u^{\prime \prime}(t)=f(t, u(t), u(0), u(1)) \\
u^{\prime}(0)=0, u^{\prime}(1)=0
\end{array}\right.
$$

Remark 4.3. We conclude the paper by showing that the method we applied in order to prove Theorems 3.1 and 3.4 can be adapted for the case where the kernel of the operator (2.2) is a nontrivial linear subspace of $\mathbb{R}^{k}$. For simplicity, we restrict our considerations to $k=2$ and the following problem:

$$
u_{i}^{\prime \prime}=f_{i}\left(t, u_{1}, u_{2}\right), \quad u_{i}^{\prime}(0)=\alpha_{i}\left[u_{i}\right], \quad u_{i}^{\prime}(1)=\beta_{i}\left[u_{i}\right], i=1,2,
$$

with $\alpha_{1}[\delta]=\beta_{1}[\delta]=0, \alpha_{1}[\gamma] \neq 1$, and $\left(1-\alpha_{2}[\gamma]\right) \beta_{2}[\delta]-\left(1-\beta_{2}[\gamma]\right) \alpha_{2}[\delta] \neq 0$. Clearly, condition (1.4) is satisfied, so (4.3) is a resonant problem. However, if either $\alpha_{2}[\delta] \neq 0$ or $\beta_{2}[\delta] \neq 0$, then (H2) does not hold and Theorem 3.1 cannot be applied. In this case, we have

$$
\operatorname{Ker} L=\{u \in \operatorname{Dom} L: u=(c, 0), c \in \mathbb{R}\}=\mathbb{R} .
$$

Thus $\operatorname{dim} \operatorname{Ker} L=1$. Moreover,

$$
\begin{aligned}
\operatorname{Im} L:= & \left\{y \in C\left([0,1], \mathbb{R}^{2}\right):\left(1-\alpha_{1}\left[\gamma_{1}\right]\right)^{-1}\left(1-\beta_{1}[\gamma]\right) \int_{0}^{1} \mathcal{K}_{A_{1}}(s) y_{1}(s) \mathrm{d} s\right. \\
& \left.+\int_{0}^{1} y_{1}(s) \mathrm{d} s-\int_{0}^{1} \mathcal{K}_{B_{1}}(s) y_{1}(s) \mathrm{d} s=0, y_{2} \in C([0,1], \mathbb{R})\right\} .
\end{aligned}
$$

It is easy to show that $L$ is Fredholm of index 0 . The projectors $P$ and $Q$ can be set as $P u=\left(u_{1}(0), 0\right)$ and

$$
\begin{aligned}
Q y= & \left(\widetilde { M } \left(\left(1-\alpha_{1}[\gamma]\right)^{-1}\left(1-\beta_{1}[\gamma]\right) \int_{0}^{1} \mathcal{K}_{A_{1}}(s) y_{1}(s) \mathrm{d} s\right.\right. \\
& \left.\left.+\int_{0}^{1} y_{1}(s) \mathrm{d} s-\int_{0}^{1} \mathcal{K}_{B_{1}}(s) y_{1}(s) \mathrm{d} s\right), 0\right)
\end{aligned}
$$

with

$$
\widetilde{M}=\left(\int_{0}^{1}\left(\left(1-\alpha_{1}[\gamma]\right)^{-1}\left(1-\beta_{1}[\gamma]\right) \mathcal{K}_{A_{1}}(s)-\mathcal{K}_{B_{1}}(s)\right) \mathrm{d} s+1\right)^{-1}
$$

Imposing appropriate assumptions upon function $f$, we can prove an existence result for problem (4.3) similar to Theorem 3.1. We omit further details.

\section{Acknowledgements}

M. Zima was partially supported by the Centre for Innovation and Transfer of Natural Science and Engineering Knowledge of University of Rzeszów. 
Open Access. This article is distributed under the terms of the Creative Commons Attribution 4.0 International License (http://creativecommons.org/licenses/by/4. $0 /$ ), which permits unrestricted use, distribution, and reproduction in any medium, provided you give appropriate credit to the original author(s) and the source, provide a link to the Creative Commons license, and indicate if changes were made.

Publisher's Note Springer Nature remains neutral with regard to jurisdictional claims in published maps and institutional affiliations.

\section{References}

[1] Amster, P.: Topological Methods in the Study of Boundary Value Problems, Universitext. Springer, New York (2014)

[2] Amster, P., Déboli, A.: A Neumann problem for a system depending on the unknown boundary values of the solution. Electron. J. Qual. Theory Differ. Equ. 2, 1-11 (2013)

[3] Boucherif, A., Al-malki, N.: Solvability of Neumann boundary value problems with Carathéodory nonlinearities. Electron. J. Differ. Equ. 51, 7 (2004)

[4] Cabada, A., Habets, P., Lois, S.: Monotone method for the Neumann problem with lower and upper solutions in the reverse order. Appl. Math. Comput. 117, 1-14 (2001)

[5] Cabada, A., Enguiça, R., López Somoza, L.: Positive solutions for second-order boundary value problems with sign changing Green's functions. Electron. J. Differ. Equ. 2017(245), 1-17 (2017)

[6] Dominguez-Pérez, M.A., Rodriguez-López, R.: Multipoint boundary value problems of Neumann type for functional differential equations. Nonlinear Anal. Real World Appl. 13(4), 1662-1675 (2012)

[7] Feltrin, G., Zanolin, F.: Existence of positive solutions in the superlinear case via coincidence degree: the Neumann and the periodic boundary value problems. Adv. Differ. Equ. 20(9-10), 937-982 (2015)

[8] Gaines, R.E., Mawhin, J.L.: Coincidence degree, and nonlinear differential equations, Lecture Notes in Mathematics, vol. 568. Springer, New York (1977)

[9] Granas, A., Guenther, R., Lee, J.: Nonlinear boundary value problems for ordinary differential equations. Dissertationes Math. (Rozprawy Mat.) 244, 128 (1985)

[10] Henderson, J., Kosmatov, N.: Positive solutions of the semipositone Neumann boundary value problem. Math. Model Anal. 20(5), 578-584 (2015)

[11] Infante, G., Pietramala, P., Tojo, F.A.F.: Non-trivial solutions of local and non-local Neumann boundary-value problems. Proc. R. Soc. Edinburgh Sect. A 146(2), 337-369 (2016)

[12] Landesman, E.M., Lazer, A.C.: Nonlinear perturbations of linear elliptic boundary value at resonance. J. Math. Mech. 19, 609-623 (1970)

[13] Margheri, A., Rebeloa, C., Torres, P.J.: On the use of Morse index and rotation numbers for multiplicity results of resonant BVPs. J. Math. Anal. Appl. 413, 660-667 (2014)

[14] Mawhin, J.: Problèmes aux Limites du Type de Neumann pour Certaines Équations Différentielles ou aux Dérivées Partielles non Linéaires, Equations 
Différentielles et Fonctionnelles non Linéaires, (Actes Conf. Internat. "EquaDiff 73", Brussels/Louvain-la-Neuve, 1973), pp. 123-134. Hermann, Paris (1973)

[15] Mawhin, J.: Topological Degree Methods in Nonlinear Boundary Value Problems, CBMS Regional Series in Math. No. 40, Amer. Math. Soc., Providence (1979)

[16] Mawhin, J., Szymańska-Dębowska, K.: Second-order ordinary differential systems with nonlocal Neumann conditions at resonance. Ann. Mat. Pura Appl. 195(5), 1605-1617 (2016)

[17] Nirenberg, L.: An application of generalized degree to a class of nonlinear problems, in Troisième Colloque du C.B.R.M. d'analyse fonctionnelle, Vander, Louvain, 57-74 (1971)

[18] Palamides, P.K.: Boundary-value problems for shallow elastic membrane caps. IMA J. Appl. Math. 67(3), 281-299 (2002)

[19] Rachůnková, I.: Upper and lower solutions and topological degree. J. Math. Anal. Appl. 234, 311-327 (1999)

[20] Rachůnková, I., Stanĕk, S.: Topological degree method in functional boundary value problems at resonance. Nonlinear Anal. 27(3), 271-285 (1996)

[21] Szymańska-Dȩbowska, K.: k-dimensional nonlocal boundary-value problems at resonance, Electron. J. Differ. Equ. no. 148, 8 pp (2015)

[22] Szymańska-Dȩbowska, K.: On the existence of solutions for nonlocal boundary value problems. Georgian Math. J. 22(2), 273-279 (2015)

[23] Szymańska-Dȩbowska, K.: On second order nonlocal boundary value problem at resonance. Fasc. Math. No. 56, 143-153 (2016)

[24] Webb, J.R.L.: Existence of positive solutions for a thermostat model. Nonlinear Anal. Real World Appl. 13(2), 923-938 (2012)

[25] Wang, F., Zhang, F.: Existence of positive solutions of Neumann boundary value problem via a cone compression-expansion fixed point theorem of functional type. J. Appl. Math. Comput. 35(1-2), 341-349 (2011)

Katarzyna Szymańska-Dȩbowska

Institute of Mathematics

Lodz University of Technology

Wólczańska 215

90-924 Łódź

Poland

e-mail: katarzyna.szymanska-debowska@p.lodz.pl

Mirosława Zima

Department of Functional Analysis, Faculty of Mathematics and Natural Sciences University of Rzeszów

Pigonia 1

35-959 Rzeszów

Poland

e-mail: mzima@ur.edu.pl 